\title{
Distracted doctoring: Smartphones before patients?
}

I stood in front of the darkened classroom, noting that many of the medical students' faces were bathed in the eerie blue glow from laptop screens. My heart sank. When we moved into this wired classroom, I complained to colleagues that students no longer seemed to listen - they were distracted by their laptops. I was told that students need their computers to take notes and I was urged to make my teaching sessions more engaging. "You must try harder." And try I did. But the laptops kept glowing.

A 39-year-old man suffering from multiple facial contusions and a head injury after a water-skiing accident was seen in the emergency department, accompanied by his spouse. The resident began taking a history, then stopped mid-sentence, pulled out his phone, read the screen and began to text. The spouse of the patient said, "What are you doing?" The resident replied, "I have to answer this. It's about dinner.' He turned his back, continued to text, waited for a response, then texted again. Replacing his phone, he started again with the history. When the spouse complained about the interruption, the resident looked at her blankly, and again stated, "I had to answer it. It was about dinner."

Medicine is a vocation of focused attention. To be a good physician, an ability to selectively identify key information is essential. Patients must be listened to and attended to, both for what they are saying and what they are choosing not to say. Generating a differential diagnosis means focusing on what is relevant and discarding what is not. These skills are at odds with the electronic multitasking learning environments we are creating for undergraduate medical students. Are we teaching students that it is acceptable to continuously interact with electronic gadgets while practising medicine?

Focused attention is difficult to promote in an age of electronic multitasking. Students sincerely believe that they can multitask and perform just as well as

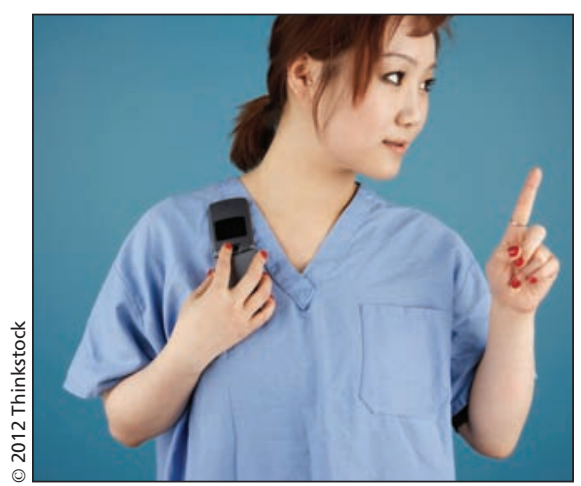

those who remain focused on one task. However, research on multitasking and divided attention consistently shows that learning and memory are impaired when there are too many distractions present. Models like Lang's Limited Processing Capacity suggest that memory can be adversely affected by interruptions and distractions. ${ }^{1,2}$ This can lead to errors or poor recall of material presented. It's critical to address the dangers of distractions by acknowledging them and by teaching students why they need to focus.

Distractions in clinical care can lead to disastrous consequences. For example, errors in medication are significantly associated with interruptions. And the severity of the medication error increases as the number of interruptions increases. Without interruption, the estimated risk of a major error is $2.3 \%$; with four interruptions, this risk doubles. ${ }^{3}$

In one study, researchers implemented a "no interruption zone" by placing red tape on the floor around the site of drug preparation to act as a visual "do not disturb" sign. With this intervention, there was a $40.9 \%$ reduction in the number of interruptions during medication preparation. ${ }^{4}$ This study reinforces the idea that changing the environment can change behaviour. The right behaviours (appropriate attention to the task at hand) did not occur naturally — the environment had to be shaped consciously.

So what are we teaching students when we enable a learning environment that encourages distractions and dis- courages focus? What are we modelling for students when we, as medical educators, are using our available "free" time to check email on our smartphones? Is this multitasking? Or is it distracted doctoring?

We are not opposed to the use of technology and e-learning. However, we are suggesting that we do students, and their future patients, a disservice when we teach and model active multitasking in clinical contexts. We propose that the learning environment be modified: use e-technologies such as wireless classrooms only for those situations where the tool is part of the active learning that is occurring.

In addition, e-etiquette should become a part of the medical school curriculum. We can no longer assume that all medical students and residents know when and where it is appropriate to use various etechnologies and communication devices.

Finally, we need to become role models. Students and residents need to see, through our behaviour, when it is appropriate to answer a call, send a text or check email.

At a recent national education conference, we glanced around the darkened room as the speaker at the podium discussed the future of medical education. Many faces of our physician colleagues were bathed in the eerie blue glow from their phones and tablets ... and we saw the opportunity to set an example for the future of medicine.

\section{Shelley Ross PhD \\ Family medicine \\ Sarah Forgie MD \\ Pediatrics \\ University of Alberta \\ Edmonton, Alta.}

For references, see Appendix 1, available at www.cmaj.ca/lookup/suppl/doi:10.1503 /cmaj.120462/-/DC1

The emergency department incident depicted here is fictitious. 